\title{
A CHARACTERIZATION OF ALGEBRAS OF INVARIANT-COINVARIANT MODULE TYPE
}

\author{
RYOHEI MAKINO
}

\begin{abstract}
K. R. Fuller has characterized rings of left invariant module type. An algebra is said to be of invariant-coinvariant module type if each of its indecomposable modules is quasi-injective or quasi-projective. In this note we shall give a characterization of algebras of invariant-coinvariant module type, which distinguishes this class from that of algebras of local-colocal type. It seems of interest that the distributivity of second radicals of primitive ideals appears in our characterization.
\end{abstract}

Throughout, $A$ denotes an artinian ring with the radical $N$ possessing a unit element 1 , and an algebra means a finite dimensional algebra over a field $P$. All modules considered are unitary $A$-modules. For an $A$-module $M$ if the socle (resp. top) of $M$ is simple, $M$ is said to be a colocal (resp. local) module. We shall say that $A$ is of left colocal (resp. local-colocal) type if every indecomposable left $A$-module is colocal (resp. local or colocal).

A ring $A$ is said to be of left invariant module type if every indecomposable left $A$-module is quasi-injective. Wu and Jans [8] showed that an indecomposable quasi-injective (resp. quasi-projective) module is colocal (resp. local). Thus, as pointed out by Dickson and Fuller [2], rings of left invariant module type are of left colocal type. Extending a result on algegras in [2] to artinian rings, Fuller [3] characterized rings of left invariant module type as those of left colocal type having the property that any primitive right ideal is distributive (i.e. its submodules form a distributive lattice (cf. Camillo [1])). In this note we shall say an algebra $A$ is of invariant-coinvariant (module) type if every indecomposable $A$-module is quasiinjective or quasi-projective. In [7] Tachikawa determined the structure of algebras of local-colocal type ([7, Theorem 4.3]). Using his structure theorem, it is not hard to show that any algebra $A$ of local-colocal type is of invariant-coinvariant type in case $N^{2}=0$. So arises the question whether or not the class of algebras of invariant-coinvariant type is properly contained in that of algebras of local-colocal type. Our theorem distinguishes algebras of invariant-coinvariant type from those of local-colocal type, and is stated as follows.

THEOREM. An algebra $A$ is of invariant-coinvariant type if and only if the following conditions are satisfied.

(I) $A$ is of local-colocal type.

(II) Each of $N^{2} e$ and $e N^{2}$ is distributive for any primitive idempotent $e$ in $A$.

Received by the editors April 5, 1983 and, in revised form, September 25, 1983.

1980 Mathematics Subject Classification. Primary 16A48, 16A64.

Key words and phrases. Algebra of local-colocal type, distributive module, quasi-injective module, quasi-projective module. 
Before proving this theorem we note the following. Let $A$ be an algebra of localcolocal type, and $M_{i}(i=1,2)$ local left $A$-modules with $N M_{i}=K_{i} \oplus L_{i}(i=1,2)$ where $K_{i}$ and $L_{i}(i=1,2)$ are serial (i.e. its composition series is unique). Then it follows from the proof of Fuller [4, Theorem 2.3] and Tachikawa [7, Proposition 3.4] that if $M_{1} / K_{1} \simeq M_{2} / K_{2}$ and $M_{1} / L_{1} \simeq M_{2} / L_{2}$, then $M_{1} \simeq M_{2}$.

PROOF OF THEOREM. Only if part: It is necessary only to show that condition (II) holds. Suppose that $e N^{2}$ is not distributive for some primitive idempotent $e$. Then by [1, Theorem 1] we can find a submodule $X$ of $e N^{2}$ such that the socle of a factor module $e N^{2} / X$ is not square free. Then since the socle of $e N / X$ is not simple, we have $e N / X=L_{1} \oplus L_{2}$ where $L_{1}$ and $L_{2}$ are serial by [7, Theorem 4.3]. Clearly it is satisfied that the length $\left|L_{1}\right|,\left|L_{2}\right| \geq 2$ and the socles $S\left(L_{1}\right)$ and $S\left(L_{2}\right)$ are isomorphic. Let $K_{1}$ and $K_{2}$ be the annihilators of $L_{1}$ and $L_{2}$ in $\left(e A / X^{*}\right)$ respectively, where $(e A / X)^{*}$ denotes the $P$-dual module of $e A / X$. Then $K_{1}$ and $K_{2}$ are serial modules with $\left|K_{1}\right|,\left|K_{2}\right| \geq 3$ and $K_{1} / N K_{1} \simeq K_{2} / N K_{2}$ since $K_{1} \simeq\left((e A / X) / L_{1}\right)^{*}$ and $K_{2} \simeq\left((e A / X) / L_{2}\right)^{*}$. Moreover it holds that $K_{1}+K_{2}=$ $(e A / X)^{*}$ and $K_{1} \cap K_{2}=S\left((e A / X)^{*}\right)$.

Assume that $\left|K_{1}\right|>\left|K_{2}\right|$. Let $t_{1}=f t_{1}$ and $t_{2}=f t_{2}$ be generators of $K_{1}$ and $K_{2}$ respectively, where $f$ is a primitive idempotent. We note that $A f$ is serial by [7, Theorem 4.3]. Put $t=t_{1}+t_{2}$; then $A t \simeq K_{1}$. Since $A t \cap S^{2}\left(K_{2}\right)$ is simple where $S^{2}\left(K_{2}\right)$ is the second socle of $K_{2}$ (i.e. $S^{2}\left(K_{2}\right) / S\left(K_{2}\right)=S\left(K_{2} / S\left(K_{2}\right)\right)$ ), considering the dual of the above note we have $K_{1}+S^{2}\left(K_{2}\right) \simeq A t+S^{2}\left(K_{2}\right)$. Obviously $t \notin K_{1}+S^{2}\left(K_{2}\right)$, and hence $K_{1}+S^{2}\left(K_{2}\right) \neq A t+S^{2}\left(K_{2}\right)$. Therefore $K_{1}+S^{2}\left(K_{2}\right)$ is not invariant by an endomorphism of its injective hull, and hence not quasiinjective by [5, Theorem 1.1]. So we obtain an indecomposable $A$-module which is neither quasi-injective nor quasi-projective. This contradicts the assumption that $A$ is of invariant-coinvariant type.

Next if $\left|K_{1}\right|=\left|K_{2}\right|$, then we have $K_{1}+S^{2}\left(K_{2}\right) \simeq K_{2}+S^{2}\left(K_{1}\right)$ by the note. Since it is clear that $K_{1}+S^{2}\left(K_{2}\right) \neq K_{2}+S^{2}\left(K_{1}\right)$, also in this case $K_{1}+S^{2}\left(K_{2}\right)$ is not quasi-injective. Similarly as the above we have a contradiction.

If part: Indecomposable modules over an algebra of local-colocal type are classified into the next four types: (i) serial, (ii) not serial, simple top and simple socle, (iii) simple top and not simple socle, (iv) simple socle and not simple top. $A$-modules of type (i) are quasi-injective or quasi-projective by [4, Proposition 2.6] and those of type (ii) are projective (and at the same time injective by [7, Proposition 3.1]). Besides $A$-modules of type (iii) and type (iv) are $P$-dual modules of each other. Thus it is sufficient to show that a left $A$-module $A e / Y$ of type (iii) is quasi-projective, where $e$ is a primitive idempotent and $Y$ is a submodule of $A e$. We note that $Y \subset N^{2} e$ because $A e / Y$ is not serial.

At first let $N e=Q \oplus R$ where $Q$ and $R$ are serial. Then $N^{2} e=N Q \oplus N R$, and there does not exist any common composition factor of $N Q$ and $N R$ by the assumption (II). Therefore every submodule of $N^{2} e$ coincides with $N^{i} Q \oplus N^{j} R$ for some $i, j \geq 1$. It follows that $Y$ is invariant by all endomorphisms of $A e$. Thus $A e / Y$ is quasi-projective.

Next if it is not the case, then $A e$ has the simple socle $S$ and $Y \supset S$. From the fact that $\mathrm{Ne} / \mathrm{S}$ is a direct sum of two serial modules, similarly as the above we can show that $Y / S$ is invariant by all endomorphisms of $A e / S$. Since $A e / S$ 
is quasi-projective, it follows that also $A e / Y \simeq(A e / S) /(Y / S)$ is quasi-projective. This completes the proof.

EXAMPLE. Let $A$ be an algebra of matrices of the form

$$
\left(\begin{array}{cccc}
x & 0 & 0 & 0 \\
a & x & 0 & 0 \\
d & b & y & 0 \\
f & e & c & z
\end{array}\right)
$$

with entries in a field $P$. Then $A$ is an algebra of right colocal type. Let $e_{i j}$ be the $i j$ th matrix unit, and $e=e_{11}+e_{22}$. As easily seen, $S\left(N^{2} e\right) \simeq A f / N f \oplus A f / N f$, where $f=e_{33}$. So $A$ is not of invariant-coinvariant type.

\section{REFERENCES}

1. V. P. Camillo, Distributive modules, J. Algebra 36 (1975), 16-25.

2. S. E. Dickson and K. R. Fuller, Algebras for which every indecomposable right module is invariant in its injective envelope, Pacific J. Math. 31 (1969), 655-658.

3. K. R. Fuller, Rings of left invariant module type, Comm. Algebra 6 (1978), 153-167.

4. __ Biserial rings, Lecture Notes in Math., vol. 734, Springer-Verlag, Berlin and New York, 1979, pp. 64-90.

5. R. E. Johnson and E. T. Wong, Quasi-injective modules and irreducible rings, J. London Math. Soc. 36 (1961), 260-268.

6. H. Tachikawa, On rings for which every indecomposable right module has a unique maximal submodule, Math. Z. 71 (1959), 200-222.

7. _ On algebras of which every indecomposable representation has an irreducible one as the top or the bottom Loewy constituent, Math. Z. 75 (1961), 215-227.

8. L. E. T. Wu and J. P. Jans, On quasi projectives, Illinois J. Math. 11 (1967), 439-448.

DEPARTMENT OF MATHEMATICS, FACUlTy OF EDUCATION, FUKUSHIMA UNiVERSITY, FUKUSHIMA 960-12, JAPAN 